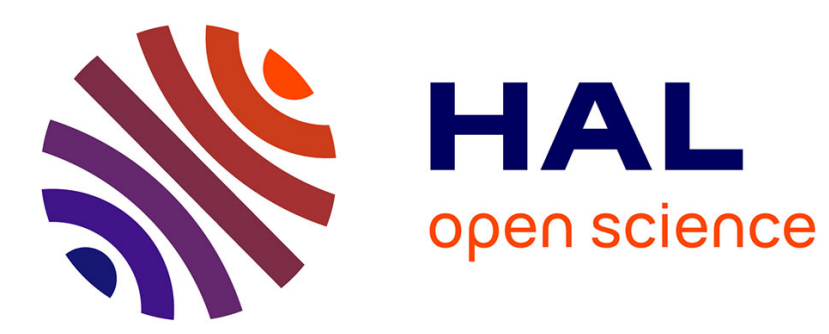

\title{
The Dirac-Witten operator on pseudo-Riemannian manifolds
}

Oussama Hijazi, Xiao Zhang, Chen Daguang

\section{To cite this version:}

Oussama Hijazi, Xiao Zhang, Chen Daguang. The Dirac-Witten operator on pseudo-Riemannian manifolds. Mathematische Zeitschrift, 2012, Math. Z., 271, pp.357-372. 10.1007/s00209-011-0867-x . hal-01270180

\section{HAL Id: hal-01270180 \\ https://hal.science/hal-01270180}

Submitted on 9 Feb 2016

HAL is a multi-disciplinary open access archive for the deposit and dissemination of scientific research documents, whether they are published or not. The documents may come from teaching and research institutions in France or abroad, or from public or private research centers.
L'archive ouverte pluridisciplinaire HAL, est destinée au dépôt et à la diffusion de documents scientifiques de niveau recherche, publiés ou non, émanant des établissements d'enseignement et de recherche français ou étrangers, des laboratoires publics ou privés. 


\title{
THE DIRAC-WITTEN OPERATOR ON PSEUDO-RIEMANNIAN MANIFOLDS
}

\author{
DAGUANG CHEN, OUSSAMA HIJAZI, AND XIAO ZHANG
}

\begin{abstract}
We study the Dirac-Witten operator for spacelike spin submanifolds in pseudo-Riemannian manifolds. When the normal bundles are spin and odd-dimensional, we derive new eigenvalue lower bounds. We also prove the generalized positive mass theorem using this operator.
\end{abstract}

\section{INTRODUCTION}

In this paper, we will study the Dirac-Witten operator of spacelike spin submanifolds in pseudo-Riemannian manifolds. In the case of spacelike hypersurfaces in 4-dimensional spacetimes, this operator was introduced by Witten to provide a new proof of the positive mass conjecture, which was originally proved by Schoen and Yau using a geometric analysis approach $[23,24,25,26,21]$. Inspired by Witten's proof, the eigenvalues of DiracWitten-type operators were estimated in [27, 20, 17, 18, 11, 19].

We first study the eigenvalues of the Dirac-Witten operator for spacelike spin submanifolds with spin normal bundles. These could be viewed as the pseudo-Riemannian or the higher codimensional analogue of the results established in $[18,11,19]$. It is surprising to note that when the dimension of the normal bundle is odd, the Dirac-Witten operator has nice properties and certain interesting eigenvalue lower bounds can be established. Inspired by $[14,13]$, we introduce a local boundary condition for this Dirac-Witten operator for submanifolds with boundary. We obtain eigenvalue lower bounds under this boundary condition when the boundary is a generalized future/past apparent horizon. Next, we study the generalized positive mass theorem for higher dimensional spacetimes with multi-time components. From [26], we know that the Dirac-Witten operator is closely related to the total energy and the total linear momentum 3-vector in 4dimensional spacetimes. We observe that a similar phenomenon occurs for higher dimensional spacetimes with odd number of time components, and in this case, the total linear momentum is an $(n, m)$-bivector. Under a generalized dominant energy condition, we close by proving the generalized positive mass theorem.

It is a purely mathematical consideration to define the generalized dominant energy condition, the generalized future/past apparent horizon, as well as the generalized total energy and total linear momentum for higher dimensional spacetimes with multi-time components. They reduce to the standard 
definitions in general relativity with 1-time component. There are two ways to generalize the Einstein field equations to the case of $m$-time components by using the tensors $R_{i j}-\frac{R}{2} g_{i j}$ and $R_{i j}-\frac{R}{m} g_{i j}$ respectively. However, no clear relationship can be found in the two cases relating these definitions to the energy-momentum tensor $T_{i j}$.

\section{Submanifolds And Spin Geometry}

2.1. Spacelike submanifolds. Let $N^{n+m}$ be an $(n+m)$-dimensional pseudoRiemannian manifold whose metric $\widetilde{g}$ has signature $(\underbrace{1, \cdots, 1}_{n}, \underbrace{-1, \cdots,-1}_{m})$. Let $M^{n}$ be an $n$-dimensional spacelike submanifold with the induced Riemannian metric $\bar{g}$. Denote by $\widetilde{\nabla}$ and $\bar{\nabla}$ the Levi-Civita connections of $N^{n+m}$ and $M^{n}$ respectively. Throughout this paper, we will agree on the following ranges of indices:

$$
1 \leq \alpha, \beta, \cdots \leq n+m ; \quad 1 \leq i, j, \cdots \leq n ; \quad n+1 \leq A, B, \cdots \leq n+m .
$$

The Einstein's summation notation is also used.

For any point $p \in M^{n}$ we consider an orthonormal basis $\left\{e_{\alpha}\right\}$ of $T_{p} N^{n+m}$ with $e_{A}$ normal and $e_{i}$ tangent to $M^{n}$. Let $\left\{\omega^{\alpha}\right\}$ be the dual basis of $\left\{e_{\alpha}\right\}$ so that the pseudo-Riemannian metric of $N^{n+m}$ is locally given by

$$
\widetilde{g}=\sum_{i=1}^{n}\left(\omega^{i}\right)^{2}-\sum_{A=n+1}^{n+m}\left(\omega^{A}\right)^{2} .
$$

The connection 1-forms $\omega_{\alpha}^{\beta}$ satisfy

$$
\widetilde{\nabla} e_{\alpha}=e_{\beta} \otimes \omega_{\alpha}^{\beta} .
$$

We have

$$
\omega_{\alpha \beta}=\widetilde{g}_{\alpha \gamma} \omega_{\beta}^{\gamma}=\varepsilon_{\alpha} \omega_{\beta}^{\alpha}, \quad \omega_{\alpha}=\widetilde{g}_{\alpha \beta} \omega^{\beta}=\varepsilon_{\alpha} \omega^{\alpha},
$$

where $\widetilde{g}_{\alpha \beta}$ are the components of the metric tensor of the manifold $N^{n+m}$ and the $\varepsilon_{\alpha}$ are given by

$$
\varepsilon_{\alpha}=\left\{\begin{aligned}
1, & \text { for } 1 \leq \alpha \leq n, \\
-1, & \text { for } \quad n+1 \leq \alpha \leq n+m .
\end{aligned}\right.
$$

Then the structure equations of $N^{n+m}$ are given by

$$
\begin{aligned}
d \omega_{\alpha} & =-\varepsilon_{\beta} \omega_{\alpha \beta} \wedge \omega_{\beta}, \quad \omega_{\alpha \beta}=-\omega_{\beta \alpha}, \\
d \omega_{\beta \alpha} & =-\varepsilon_{\gamma} \omega_{\beta \gamma} \wedge \omega_{\gamma \alpha}+\frac{1}{2} \varepsilon_{\gamma} \varepsilon_{\delta} \widetilde{R}_{\beta \alpha \gamma \delta} \omega_{\gamma} \wedge \omega_{\delta} .
\end{aligned}
$$


The curvature tensor $\widetilde{R}_{\alpha \beta \gamma \delta}$, the Ricci tensor $\widetilde{R}_{\alpha \beta}$ and the scalar curvature $\widetilde{R}$ of $N$ are given by

$$
\begin{aligned}
\widetilde{R}(X, Y) Z & =\widetilde{\nabla}_{X} \widetilde{\nabla}_{Y} Z-\widetilde{\nabla}_{Y} \widetilde{\nabla}_{X} Z-\widetilde{\nabla}_{[X, Y]} Z, \quad X, Y, Z \in \Gamma(T N) ; \\
\widetilde{R}_{\alpha \beta \gamma \delta} & =\widetilde{g}\left(\widetilde{R}\left(e_{\gamma}, e_{\delta}\right) e_{\beta}, e_{\alpha}\right)=\widetilde{g}_{\alpha \zeta} \widetilde{R}_{\beta \gamma \delta} ; \\
\widetilde{R} i c(X, Y) & =\varepsilon_{\alpha} \widetilde{g}\left(\widetilde{R}\left(e_{\alpha}, X\right) Y, e_{\alpha}\right), \\
\widetilde{R}_{\alpha \beta} & =\widetilde{R}_{\alpha \gamma \beta}=\varepsilon_{\gamma} \widetilde{R}_{\alpha \gamma \beta \gamma} ; \\
\widetilde{R} & =\varepsilon_{\alpha} \widetilde{R}\left(e_{\alpha}, e_{\alpha}\right)=\widetilde{g}^{\alpha \beta} \widetilde{R}_{\alpha \beta}=\varepsilon_{\alpha} \varepsilon_{\gamma} \widetilde{R}_{\alpha \gamma \alpha \gamma} .
\end{aligned}
$$

The curvature tensor $\bar{R}_{i j k l}$, the Ricci tensor $\bar{R}_{i j}$ and the scalar curvature $\bar{R}$ of $M^{n}$ can be defined in a similar way. From submanifold theory, the Gauss, the Ricci and the Codazzi equations for the spacelike submanifold $M^{n}$ give rise, respectively to

$$
\begin{aligned}
\widetilde{R}_{i j k l} & =\bar{R}_{i j k l}+\left(p_{A i k} p_{A j l}-p_{A i l} p_{A j k}\right) \\
\widetilde{R}_{A B k l} & =\bar{R}_{A B k l}-\left(p_{A i k} p_{B i l}-p_{A i l} p_{B i k}\right) \\
\widetilde{R}_{i A k j} & =p_{A j i k}-p_{A i k j}
\end{aligned}
$$

where $p_{A i j}:=\widetilde{g}\left(\widetilde{\nabla}_{i} e_{A}, e_{j}\right)$ are the components of the second fundamental form of $M^{n}$, and $p_{A i j k}$ the covariant derivative of $p_{A i j}$, are defined by

$$
p_{A i j k} \omega_{k}=d p_{A i j}-p_{A k j} \omega_{k i}-p_{A i k} \omega_{k j}+p_{B i j} \omega_{B A} .
$$

2.2. Spin connection. Let $C l_{n, m}$ be the Clifford algebra with respect to the above pseudo-Riemannian metric $\widetilde{g}$. (We refer to Baum $[5,6]$ for a detailed algebraic constructions.) For any vector field $X \in \Gamma\left(T N^{n+m}\right)$,

$$
\left(e_{\alpha} \wedge e_{\beta}\right) X=\widetilde{g}\left(e_{\alpha}, X\right) e_{\beta}-\widetilde{g}\left(e_{\beta}, X\right) e_{\alpha},
$$

where $e_{\alpha} \wedge e_{\beta}$ is the canonical basis of the Lie algebra $\mathfrak{s o}(n, m)$. The LeviCivita connection on a pseudo-Riemannian manifold $N^{n+m}$ induces the connection on the principle $\mathrm{SO}(n, m)$-bundle with the connection 1-form

$$
\omega=-\frac{1}{2} \varepsilon_{\alpha} \varepsilon_{\beta} \omega_{\alpha \beta} e_{\alpha} \wedge e_{\beta} .
$$

The (local) spinorial connection of $N^{n+m}$ is lifted form the principle $\mathrm{SO}(n, m)$ connection as

$$
\widetilde{\nabla} \varphi=d \varphi-\frac{1}{4} \varepsilon_{\alpha} \varepsilon_{\beta} \omega_{\alpha \beta} e_{\alpha} e_{\beta} \varphi .
$$

Suppose that $M^{n}$ is a spin submanifold whose normal bundle $\xi$ in $N^{n+m}$ is also spin. Denote by $K$ the maximal compact subgroup of $\operatorname{Spin}_{0}(n, m)$, the connected component of the spin group. Let $\$$ be the (local) spinor bundle of $N^{n+m}$. 
Since the following diagram is commutative

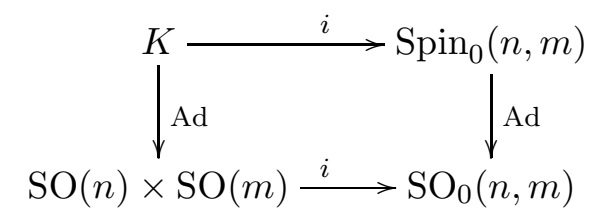

the induced spinor bundle $\mathbb{S}:=\left.\$\right|_{M^{n}}$ is globally defined over $M^{n}$.

Denote also by $\widetilde{\nabla}$ and $\bar{\nabla}$ the spin connections on $\mathbb{S}$. It is well-known $[5,6]$ that there exists a Hermitian inner product $($,$) on \$$ which is compatible with the spin (local) connection $\widetilde{\nabla}$. Moreover, for any vector field $X \in$ $\Gamma\left(T N^{n+m}\right)$ and spinor fields $\varphi, \psi \in \Gamma(\$)$,

$$
(X \varphi, \psi)=(\varphi, X \psi)
$$

locally. Note that this inner product is not positive definite and all these data are global over $M^{n}$.

The above diagram also implies that, over $M^{n}$, there exists a positive definite Hermitian inner product on $\mathbb{S}$ which is defined as

$$
\langle,\rangle=(\omega,)
$$

where $\omega=(\sqrt{-1})^{\frac{m(m-1)}{2}} e_{n+1} \cdots e_{n+m}$ is the complex volume element of the normal bundle over $M^{n}$. Obviously $\omega^{2}=1$ and we have

$$
(\omega \varphi, \omega \psi)=(\varphi, \psi)
$$

which implies

$$
\langle\omega \varphi, \psi\rangle=\langle\varphi, \omega \psi\rangle
$$

Lemma 2.1. Suppose that $M^{n}$ is a spin submanifold whose normal bundle is also spin. Over $M^{n}$, we have

(i) If $m$ is odd, then $\omega e_{i}=-e_{i} \omega, \omega e_{A}=e_{A} \omega$. Moreover,

$$
\left\langle e_{i} \varphi, \psi\right\rangle=-\left\langle\varphi, e_{i} \psi\right\rangle, \quad\left\langle e_{A} \varphi, \psi\right\rangle=\left\langle\varphi, e_{A} \psi\right\rangle .
$$

(ii) If $m$ is even, then $\omega e_{i}=e_{i} \omega, \omega e_{A}=-e_{A} \omega$. Moreover,

$$
\left\langle e_{i} \varphi, \psi\right\rangle=\left\langle\varphi, e_{i} \psi\right\rangle, \quad\left\langle e_{A} \varphi, \psi\right\rangle=-\left\langle\varphi, e_{A} \psi\right\rangle .
$$

Defining the second fundamental form of $M^{n}$ by

$$
p_{A i j}=\widetilde{g}\left(\widetilde{\nabla}_{i} e_{A}, e_{j}\right)=\omega_{j A}\left(e_{i}\right),
$$

we have the following spinorial Gauss formula

$$
\widetilde{\nabla}_{i} \varphi=\bar{\nabla}_{i} \varphi+\frac{1}{2} \omega_{A j}\left(e_{i}\right) e_{A} e_{j} \varphi=\bar{\nabla}_{i} \varphi+\frac{1}{2} p_{A i j} e_{j} e_{A} \varphi .
$$

Lemma 2.2. Suppose that $M^{n}$ is a spin submanifold whose normal bundle is also spin. For the normal volume element $\omega$, we have

$$
\widetilde{\nabla}_{i} \omega=p_{A i j} e_{j} e_{A} \omega \text {. }
$$


Proof. Note that

$$
\widetilde{\nabla}_{i} e_{n+r}=p_{n+r, i j} e_{j}-\sum_{1 \leq s \leq m} \omega_{n+s, n+r}\left(e_{i}\right) e_{n+s},
$$

we obtain

$$
\begin{aligned}
\widetilde{\nabla}_{i} \omega= & (\sqrt{-1})^{\frac{m(m-1)}{2}} \widetilde{\nabla}_{i}\left(e_{n+1} \cdots e_{n+m}\right) \\
= & (\sqrt{-1})^{\frac{m(m-1)}{2}} \sum_{1 \leq r \leq m} e_{n+1} \cdots \widetilde{\nabla}_{i} e_{n+r} \cdots e_{n+m} \\
= & (\sqrt{-1})^{\frac{m(m-1)}{2}}\left(\sum_{1 \leq r \leq m} p_{n+r, j i}(-1)^{r-1} e_{j} e_{n+1} \cdots \hat{e}_{n+r} \cdots e_{n+m}\right. \\
& -\sum_{1 \leq r<s \leq m} \omega_{n+s, n+r}\left(e_{i}\right) e_{n+1} \cdots \stackrel{(r)}{e}_{n+s} \cdots \stackrel{(s)}{e}_{n+s} \cdots e_{n+m} \\
& \left.-\sum_{1 \leq s<r \leq m} \omega_{n+s, n+r}\left(e_{i}\right) e_{n+1} \cdots \stackrel{(s)}{e}_{n+s} \cdots \stackrel{(r)}{e}_{n+s} \cdots e_{n+m}\right) \\
= & (\sqrt{-1})^{\frac{m(m-1)}{2}}\left(\sum_{1 \leq r \leq m} p_{n+r, j i}(-1)^{r-1} e_{j} e_{n+1} \cdots \hat{e}_{n+r} \cdots e_{n+m}\right. \\
& +\sum_{1 \leq r<s \leq m}(-1)^{s-r} \omega_{n+s, n+r}\left(e_{i}\right) e_{n+1} \cdots \hat{e}_{n+r} \cdots \hat{e}_{n+s} \cdots e_{n+m} \\
& \left.+\sum_{1 \leq s<r \leq m}(-1)^{r-s} \omega_{n+s, n+r}\left(e_{i}\right) e_{n+1} \cdots \hat{e}_{n+s} \cdots \hat{e}_{n+r} \cdots e_{n+m}\right) \\
= & (\sqrt{-1})^{\frac{m(m-1)}{2}} \sum_{1 \leq r \leq m} p_{n+r, j i}(-1)^{r-1} e_{j} e_{n+1} \cdots \hat{e}_{n+r} \cdots e_{n+m} \\
= & p_{A j i} e_{j} e_{A} \omega .
\end{aligned}
$$

Lemma 2.3. Suppose that $M^{n}$ is a spin submanifold whose normal bundle is also spin. The connection $\bar{\nabla}$ is compatible with $\langle$,$\rangle .$

Proof. Since $\omega e_{j} e_{A}=-e_{j} e_{A} \omega$ for any $m$, we obtain

$$
\begin{aligned}
e_{i}\langle\varphi, \psi\rangle= & e_{i}(\omega \varphi, \psi) \\
= & \left(\widetilde{\nabla}_{i} \omega \varphi, \psi\right)+\left(\omega \widetilde{\nabla}_{i} \varphi, \psi\right)+\left(\omega \varphi, \widetilde{\nabla}_{i} \psi\right) \\
= & \left(p_{A i j} e_{j} e_{A} \omega \varphi, \psi\right)+\left\langle\bar{\nabla}_{i} \varphi, \psi\right\rangle+\frac{1}{2}\left(p_{A i j} \omega e_{j} e_{A} \varphi, \psi\right) \\
& +\left\langle\varphi, \bar{\nabla}_{i} \psi\right\rangle+\frac{1}{2}\left(\omega \varphi, p_{A i j} e_{j} e_{A} \psi\right) \\
= & \left\langle\bar{\nabla}_{i} \varphi, \psi\right\rangle+\left\langle\varphi, \bar{\nabla}_{i} \psi\right\rangle .
\end{aligned}
$$

Let $N^{n+m}$ be an $(n+m)$-dimensional pseudo-Riemannian manifold and $M^{n}$ an $n$-dimensional spin spacelike submanifold of $N^{n+m}$. Suppose that 
the normal bundle of $M^{n}$ is also spin. The Dirac-Witten operator over $M^{n}$ is defined as

$$
\widetilde{D}=\sum_{i} e_{i} \widetilde{\nabla}_{i}
$$

The intrinsic Dirac operator of $M^{n}$ acting on $\mathbb{S}$ is defined as

$$
\bar{D}=\sum_{i} e_{i} \bar{\nabla}_{i}
$$

The relation between the operators $\widetilde{D}$ and $\bar{D}$ is given by

$$
\widetilde{D}=e_{i}\left(\bar{\nabla}_{i}+\frac{1}{2} p_{A i j} e_{j} e_{A}\right)=\bar{D}-\frac{1}{2} P_{A} e_{A}
$$

where $P_{A}=\sum_{i=1}^{n} p_{A i i}$.

Proposition 2.4. Suppose that $M^{n}$ is a compact manifold without boundary.

(i) The Dirac operator $\bar{D}$ is formally self-adjoint with respect to the positive definite $L^{2}$ inner product $\int_{M}\langle$,$\rangle ;$

(ii) The Dirac-Witten operator $\widetilde{D}$ is formally self-adjoint with respect to this inner product if the codimension of $M^{n}$ is odd.

Proof. A straightforward calculation yields

$$
\begin{aligned}
e_{i}\left\langle e_{i} \varphi, \psi\right\rangle & =\left\langle\bar{\nabla}_{i} e_{i} \varphi, \psi\right\rangle+\left\langle e_{i} \bar{\nabla}_{i} \varphi, \psi\right\rangle+\left\langle e_{i} \varphi, \bar{\nabla}_{i} \psi\right\rangle \\
& =\left\langle\bar{\nabla}_{i} e_{i} \varphi, \psi\right\rangle+\langle\bar{D} \varphi, \psi\rangle-\langle\varphi, \bar{D} \psi\rangle .
\end{aligned}
$$

Define the vector field $X=\left\langle e_{i} \varphi, \psi\right\rangle e_{i}$, then

$$
\begin{aligned}
\operatorname{div} X & =\bar{g}\left(\bar{\nabla}_{i} X, e_{i}\right) \\
& =\bar{g}\left(\bar{\nabla}_{i}\left(\left\langle e_{j} \varphi, \psi\right\rangle e_{j}\right), e_{i}\right) \\
& =e_{i}\left\langle e_{i} \varphi, \psi\right\rangle-\left\langle\bar{\nabla}_{i} e_{i} \varphi, \psi\right\rangle \\
& =e_{i}\left\langle e_{i} \varphi, \psi\right\rangle-\left\langle\bar{\nabla}_{i} e_{i} \varphi, \psi\right\rangle .
\end{aligned}
$$

Therefore the Dirac operator $\bar{D}$ is formally self-adjoint. By the relation between $\bar{D}$ and $\widetilde{D}$, it follows

$$
\operatorname{div} X=\langle\widetilde{D} \varphi, \psi\rangle-\langle\varphi, \widetilde{D} \psi\rangle+\frac{P_{A}}{2}\left\langle e_{A} \varphi, \psi\right\rangle-\frac{P_{A}}{2}\left\langle\varphi, e_{A} \psi\right\rangle
$$

For $m$ odd, the sum of the last two terms vanishes, which implies that $\widetilde{D}$ is formally self-adjoint.

Now we derive the following Weitzenböck type formula.

Theorem 2.5. If the codimension of $M^{n}$ is odd, then

$$
\widetilde{D}^{2}=\widetilde{\nabla}^{*} \widetilde{\nabla}+\frac{1}{4} \sum_{i, j} \widetilde{R}_{i j i j}-\frac{1}{2} \sum_{i, j, A} \widetilde{R}_{i j A j} e_{i} e_{A}+\frac{1}{4} \sum_{\{\alpha, \beta\} \neq\{i, A\} ; \alpha<\beta} \widetilde{R}_{i A \alpha \beta} e_{i} e_{A} e_{\alpha} e_{\beta},
$$

where $\widetilde{\nabla}_{j}^{*}=-\widetilde{\nabla}_{j}+p_{A i j} e_{i} e_{A} \varphi$ is the formal adjoint of $\widetilde{\nabla}$. 
Proof. It is straightforward that

$$
\begin{aligned}
& \widetilde{D}^{2} \varphi=e_{i} \widetilde{\nabla}_{i}\left(e_{j} \widetilde{\nabla}_{j} \varphi\right) \\
& =e_{i} \widetilde{\nabla}_{i} e_{j} \widetilde{\nabla}_{j} \varphi+e_{i} e_{j} \widetilde{\nabla}_{i} \widetilde{\nabla}_{j} \varphi \\
& =p_{A i j} e_{i} e_{A} \widetilde{\nabla}_{j} \varphi+\frac{1}{2} e_{i} e_{j}\left(\widetilde{\nabla}_{i} \widetilde{\nabla}_{j}-\widetilde{\nabla}_{j} \widetilde{\nabla}_{i}\right) \varphi-\widetilde{\nabla}_{i} \widetilde{\nabla}_{i} \varphi \\
& =\widetilde{\nabla}^{*} \widetilde{\nabla} \varphi-\frac{1}{8} \widetilde{R}_{i j \alpha \beta} e_{i} e_{j} e_{\alpha} e_{\beta} \varphi \\
& =\widetilde{\nabla}^{*} \widetilde{\nabla} \varphi-\frac{1}{8} \sum_{\gamma \neq \alpha \neq \beta} \widetilde{R}_{i \gamma \alpha \beta} e_{i} e_{\gamma} e_{\alpha} e_{\beta} \varphi-\frac{1}{8} \widetilde{R}_{i \alpha \alpha \beta} e_{i} e_{\alpha} e_{\alpha} e_{\beta} \varphi \\
& -\frac{1}{8} \widetilde{R}_{i \beta \alpha \beta} e_{i} e_{\beta} e_{\alpha} e_{\beta} \varphi+\frac{1}{8} \widetilde{R}_{i A \alpha \beta} e_{i} e_{A} e_{\alpha} e_{\beta} \varphi \\
& =\widetilde{\nabla}^{*} \widetilde{\nabla} \varphi-\frac{1}{4} \widetilde{R}_{i \beta \alpha \beta} e_{i} e_{\alpha} g^{\beta \beta} \varphi+\frac{1}{8} \widetilde{R}_{i A i \beta} e_{i} e_{A} e_{i} e_{\beta} \varphi \\
& +\frac{1}{8} \widetilde{R}_{i A A \beta} e_{i} e_{A} e_{A} e_{\beta} \varphi+\frac{1}{8} \sum_{\{\alpha, \beta\} \neq\{i, A\}} \widetilde{R}_{i A \alpha \beta} e_{i} e_{A} e_{\alpha} e_{\beta} \varphi \\
& =\widetilde{\nabla}^{*} \widetilde{\nabla} \varphi-\frac{1}{4} \widetilde{R}_{i \beta \alpha \beta} e_{i} e_{\alpha} g^{\beta \beta} \varphi \\
& -\frac{1}{8} \widetilde{R}_{i A i \beta} e_{A} e_{\beta} g^{i i} \varphi+\frac{1}{8} \widetilde{R}_{B i B \beta} e_{i} e_{\beta} g^{B B} \varphi \\
& +\frac{1}{8} \sum_{\{\alpha, \beta\} \neq\{i, A\}} \widetilde{R}_{i A \alpha \beta} e_{i} e_{A} e_{\alpha} e_{\beta} \varphi \\
& =\widetilde{\nabla}^{*} \widetilde{\nabla} \varphi+\frac{1}{4} \widetilde{R}_{i \beta i \beta} g^{i i} g^{\beta \beta} \varphi-\frac{1}{4} \widetilde{R}_{i \beta A \beta} e_{i} e_{A} g^{\beta \beta} \varphi \\
& -\frac{1}{8} \widetilde{R}_{i A i A} g^{A A} g^{i i} \varphi-\frac{1}{4} \widetilde{R}_{i A i j} e_{A} e_{j} g^{i i} \varphi \\
& -\frac{1}{8} \widetilde{R}_{B i B i} g^{i i} g^{B B} \varphi-\frac{1}{4} \widetilde{R}_{B i B A} e_{i} e_{A} g^{B B} \varphi \\
& +\frac{1}{8} \sum_{\{\alpha, \beta\} \neq\{i, A\}} \widetilde{R}_{i A \alpha \beta} e_{i} e_{A} e_{\alpha} e_{\beta} \varphi \\
& =\widetilde{\nabla}^{*} \widetilde{\nabla} \varphi+\frac{1}{4} \sum_{i, j} \widetilde{R}_{i j i j} \varphi-\frac{1}{2} \sum_{i, j, A} \widetilde{R}_{i j A j} e_{i} e_{A} \varphi \\
& +\frac{1}{4} \sum_{\{\alpha, \beta\} \neq\{i, A\} ; \alpha<\beta} \widetilde{R}_{i A \alpha \beta} e_{i} e_{A} e_{\alpha} e_{\beta} \varphi .
\end{aligned}
$$

Gauss and Codazzi equations (2) imply

$$
\sum_{i, j} \widetilde{R}_{i j i j}=\mu, \quad \sum_{j} \widetilde{R}_{i j A j}=\varpi_{i A}
$$


where

$$
\mu=\frac{1}{2}\left(\bar{R}+\left(\operatorname{Tr} p_{A}\right)^{2}-\left|p_{A}\right|^{2}\right), \quad \varpi_{i A}=\bar{\nabla}^{j} p_{A j i}-\bar{\nabla}_{i} \operatorname{Tr} p_{A} .
$$

Therefore (3) can be written as

$$
\widetilde{D}^{2}=\widetilde{\nabla}^{*} \widetilde{\nabla}+\frac{1}{2}\left(\mu-\sum_{i, A} \varpi_{i A} e_{i} e_{A}\right)+\frac{1}{4} \sum_{\{\alpha, \beta\} \neq\{i, A\} ; \alpha<\beta} \widetilde{R}_{i A \alpha \beta} e_{i} e_{A} e_{\alpha} e_{\beta} .
$$

We say that $M^{n}$ satisfies the generalized dominant energy condition if

$$
\mu \geq U:=\sqrt{\sum_{i, A} \varpi_{i A}^{2}}+\frac{1}{2} \sqrt{\sum_{\{\alpha, \beta\} \neq\{i, A\} ; \alpha<\beta} \widetilde{R}_{i A \alpha \beta}^{2}} .
$$

Theorem 2.6. Let $M^{n}$ be a compact spacelike spin submanifold of a pseudoRiemannian manifold $N^{n+m}$. Suppose that the normal bundle of $M^{n}$ is spin and odd-dimensional. Let $\lambda$ be any eigenvalue of the Dirac-Witten operator $\widetilde{D}$ with a corresponding eigenspinor $\phi$. If the generalized dominant energy condition (5) holds, then

$$
\lambda^{2} \geq \frac{n}{2(n-1)} \inf _{M}(\mu-U) .
$$

Proof. Note that (4) gives

$$
\int_{M}|\widetilde{D} \varphi|^{2} \geq \int_{M}|\widetilde{\nabla} \varphi|^{2}+\frac{1}{2}(\mu-U)|\varphi|^{2} .
$$

Define the modified connection [10]

$$
\widetilde{\nabla}_{i}^{\lambda}=\widetilde{\nabla}_{i}+\frac{\lambda}{n} e_{i}
$$

For the eigenspinor $\varphi$ corresponding to the eigenvalue $\lambda$, we have

$$
\left|\widetilde{\nabla}^{\lambda} \varphi\right|^{2}=|\widetilde{\nabla} \varphi|^{2}-\frac{\lambda^{2}}{n}|\varphi|^{2}
$$

This together with (7) implies (6). If equality holds, then (7) implies that $\widetilde{\nabla}^{\lambda} \varphi=0$ and $\mu-U$ is a non-negative constant.

\section{Boundary VALUE PROBlems}

Let $F$ be a Hermitian vector bundle over a Riemannian manifold $M^{n}$ with nonempty boundary $\Sigma$ and let $\mathcal{D}$ be a first order elliptic operator acting on the vector bundle $F$. An elliptic boundary condition for $\mathcal{D}$ can be defined as follows.

The Calderón projector is defined as

$$
\mathcal{P}_{+}(\mathcal{D}): H^{\frac{1}{2}}\left(\left.F\right|_{\Sigma}\right) \longrightarrow\left\{\left.\psi\right|_{\Sigma}: \psi \in H^{1}(F), \mathcal{D} \psi=0\right\}
$$

where $H^{s}$ is the Sobolev space. It is well known that $\mathcal{P}_{+}(\mathcal{D})$ is a pseudodifferential operator of order zero. Although the Calderón projector is not 
unique, its principal symbol $\mathbf{p}_{+}(\mathcal{D})$ is uniquely determined by the principal symbol $\sigma_{\mathcal{D}}$

$$
\mathbf{p}_{+}(\mathcal{D})(u)=-\frac{1}{2 \pi \sqrt{-1}} \int_{\Gamma}\left[\left(\sigma_{\mathcal{D}}(\nu)\right)^{-1} \sigma_{\mathcal{D}}(u)-\zeta I\right]^{-1} d \zeta
$$

for any point $p \in \Sigma$ and $u \in T_{p} \Sigma$, where $\nu$ is the inner unit normal along the boundary $\Sigma$ and $\Gamma$ is any simple closed contour oriented clockwise and enclosing all poles of the integrand in $\Im \zeta<0$. Then, an elliptic boundary condition can be defined in terms of $\mathbf{p}_{+}(\mathcal{D})[22,7]$. We refer to $[4,1]$ for the general discussion of the boundary value problems for Dirac-type operators.

Definition 3.1. A pseudo-differential operator

$$
B: L^{2}\left(\left.F\right|_{\Sigma}\right) \longrightarrow L^{2}(V)
$$

where $V \longrightarrow \Sigma$ is a complex vector bundle over the boundary, is called a (global) elliptic boundary condition if its principal symbol

$$
b: T \Sigma \longrightarrow \operatorname{Hom}_{\mathbb{C}}\left(\left.F\right|_{\Sigma}, V\right)
$$

satisfies, for any non-trivial $u \in T_{p} \Sigma, p \in \Sigma$, that the restriction

$$
\left.b(u)\right|_{\text {Image }} \mathbf{p}_{+}(\mathcal{D})(u): \text { Image } \mathbf{p}_{+}(\mathcal{D})(u) \subset F_{p} \longrightarrow V_{p}
$$

is an isomorphism onto the image $b(u) \subset V_{p}$. Moreover, if rank $V=\operatorname{dim}$ Image $\mathbf{p}_{+}(\mathcal{D})(u)$, we say that $B$ is a local elliptic boundary condition.

In this case we say that

$$
\left\{\begin{aligned}
\mathcal{D} \psi=\chi & \text { in } M^{n}, \\
\left.B \psi\right|_{\Sigma}=\phi & \text { on } \Sigma
\end{aligned}\right.
$$

is an elliptic boundary problem. An elliptic boundary problem such as (EBP) has a solution $\psi \in H^{1}(F)$ for any pair $(\chi, \phi)$ in a subspace of $L^{2}(F) \times$ $H^{1 / 2}(V)$ of finite codimension. Moreover, this solution is unique up to a finite-dimensional kernel, i.e., (EBP) is of Fredholm type.

Now we use the argument in $[15,13]$ to study an elliptic boundary problem on the spinor bundle. Let $M^{n}$ be a compact spacelike spin submanifold of pseudo-Riemannian manifold $N^{n+m}$ with spin normal bundle. Suppose $M^{n}$ has a nonempty boundary $\Sigma$ endowed with an induced Riemannian and spin structures. For any $p \in \Sigma$, we choose the orthonormal basis $\left\{e_{\alpha}\right\}_{\alpha=1}^{n+m}$ such that $e_{i}(1 \leq i \leq n)$ tangent to $M^{n}$ with $e_{n}$ the outer normal to the boundary $\Sigma, e_{a}(1 \leq a \leq n-1)$ tangent to $\Sigma$ and as before $e_{A}(n+1 \leq A \leq n+m)$ normal to $M^{n}$. Denote the boundary projection operator by

$$
B_{ \pm}=\frac{1}{2}\left(I \pm e_{n} \omega\right) \text {. }
$$

Proposition 3.1. If $m$ is odd, then the following boundary problem for the Dirac-Witten operator

$$
\left\{\begin{aligned}
\widetilde{D} \psi & =\lambda \psi, \quad \text { in } M^{n} \\
\left.B_{ \pm} \psi\right|_{\Sigma} & =0,
\end{aligned}\right.
$$


is elliptic. Moreover, (9) has a discrete spectrum with finite dimensional eigenspaces consisting of smooth spinor fields, unless it is the whole complex plane.

Proof. Without loss of generality, we prove the theorem only for the boundary operator $B_{+}$. Obviously, the symbol of the Dirac-Witten operator can be calculated by

$$
\sigma_{\widetilde{D}}(v)=\sqrt{-1} v, \quad \forall v \in T M^{n} .
$$

By (8), the principal symbol $\mathbf{p}_{+}(\widetilde{D})$ of the Calderón projector of the DiracWitten operator is given by

$$
\mathbf{p}_{+}(\widetilde{D})(u)=-\frac{1}{2|u|}\left(\sqrt{-1}\left(-e_{n}\right) u-|u| I\right), \quad \text { for } u \in T \Sigma .
$$

¿From Proposition 1 in [13], we have

$$
\begin{gathered}
\text { Image } \mathbf{p}_{+}(\widetilde{D})(u)=\left\{\psi \in \mathbb{S}: \sqrt{-1}\left(-e_{n}\right) u \psi=-|u| \psi\right\}, \\
\operatorname{dim} \operatorname{Image}_{+}(\widetilde{D})(u)=\frac{1}{2} \operatorname{dim} \mathbb{S} .
\end{gathered}
$$

Since the eigenspaces of $e_{n} \omega$ corresponding to the eigenvalues 1 and -1 are interchanged by $e_{n}$, we have

$$
\operatorname{rank} V=\frac{1}{2} \operatorname{dim} \mathbb{S} .
$$

Since $B_{+}$is a pseudo-differential operator of order zero, its principal symbol $b_{+}(u)$, on each vector $u \in T \Sigma$, coincides with the operator itself, that is,

$$
b_{+}(u)=\frac{1}{2}\left(I+e_{n} \omega\right), \quad \forall u \in T \Sigma .
$$

If $b_{+}(u) \varphi=0$, i.e. $e_{n} \omega \varphi=-\varphi$, then

$$
\begin{aligned}
\sqrt{-1} e_{n} u \varphi & =-\sqrt{-1} e_{n} u\left(e_{n} \omega\right) \varphi \\
& =e_{n} \omega\left(\sqrt{-1} e_{n} \omega \varphi\right) .
\end{aligned}
$$

This implies that $\sqrt{-1} e_{n} u \varphi$ belongs to the positive eigenspace of $e_{n} \omega$. Therefore,

$$
\text { Ker } b_{+}(u) \cap \text { Image } \mathbf{p}_{+}(\widetilde{D})(u)=\{0\} .
$$

¿From (10) and (11), the elliptic boundary condition for the pseudo-differential operator $B_{+}$are satisfied by Proposition 1 in [13]. The remaining assertions on eigenvalues and eigenspaces are straightforward (see $[7,16]$ ).

For any point $p \in \Sigma$ and an orthonormal basis $\left\{e_{i}\right\}$ of $T_{p} M^{n}$ with $e_{n}$ the outward normal to $\Sigma$ and $e_{a}$ tangent to $\Sigma$ for $1 \leq a \leq n-1$. Let

$$
h_{a b}=\bar{g}\left(\bar{\nabla}_{a} e_{n}, e_{b}\right)
$$

be the components of the second fundamental form of $\Sigma$. Let $H=\sum_{a=1}^{n-1} h_{a a}$ be its mean curvature. 
Denote by $\nabla$ the lift of the Levi-Civita connection of $\Sigma$ to the spinor bundle $\left.\mathbb{S}\right|_{\Sigma}$. Then the spinorial Gauss formula is given by

$$
\bar{\nabla}_{a}=\nabla_{a}+\frac{1}{2} h_{a b} e_{n} e_{b}
$$

The Dirac operator $D$ of $\Sigma$ acting on $\left.\mathbb{S}\right|_{\Sigma}$ is defined as

$$
D=e_{a} \nabla_{a}
$$

A straightforward calculation yields the connection $\nabla$ is also compatible with the Hermitian metric $\langle$,$\rangle on the spinor bundle over the boundary \Sigma$. Moreover,

$$
\widetilde{\nabla}_{a}=\nabla_{a}+\frac{1}{2} h_{a b} e_{n} e_{b}+\frac{1}{2} p_{A a j} e_{j} e_{A} .
$$

Lemma 3.2. The following identities hold

$$
\nabla_{a}\left(e_{n} \varphi\right)=e_{n} \nabla_{a} \varphi, \quad \nabla_{a}\left(e_{A} \varphi\right)=e_{A} \nabla_{a} \varphi
$$

Proof.

$$
\begin{aligned}
\nabla_{a}\left(e_{n} \varphi\right)= & \left(\bar{\nabla}_{a}-\frac{1}{2} h_{a b} e_{n} e_{b}\right)\left(e_{n} \varphi\right) \\
= & \bar{\nabla}_{a}\left(e_{n} \varphi\right)-\frac{1}{2} h_{a b} e_{n} e_{b} e_{n} \varphi \\
= & \bar{\nabla}_{a} e_{n} \varphi+e_{n} \bar{\nabla}_{a} \varphi-\frac{1}{2} h_{a b} e_{b} \varphi \\
= & h_{a b} e_{b} \varphi+\left(e_{n} \nabla_{a}+\frac{1}{2} h_{a b} e_{n} e_{b}\right) \varphi-\frac{1}{2} h_{a} b e_{b} \varphi \\
= & e_{n} \nabla_{a} \varphi \\
\nabla_{a}\left(e_{A} \varphi\right)= & \left(\widetilde{\nabla}_{a}-\frac{1}{2} h_{a b} e_{n} e_{b}-\frac{1}{2} p_{B a j} e_{j} e_{B}\right)\left(e_{A} \varphi\right) \\
= & \widetilde{\nabla}_{a}\left(e_{A} \varphi\right)-\frac{1}{2} h_{a b} e_{n} e_{b} e_{A} \varphi-\frac{1}{2} p_{B a j} e_{j} e_{B} e_{A} \varphi \\
= & p_{A a j} e_{j} \varphi+e_{A}\left(\nabla_{a}+\frac{1}{2} h_{a b} e_{n} e_{b}+\frac{1}{2} p_{B a j} e_{j} e_{B}\right) \varphi \\
& -\frac{1}{2} h_{a b} e_{n} e_{b} e_{A} \varphi-\frac{1}{2} p_{B a j} e_{j} e_{B} e_{A} \varphi \\
= & e_{A} \nabla_{a} \varphi
\end{aligned}
$$

Lemma 3.3. The Dirac operator acting on the spinor bundle $\mathbb{S}$ and the local boundary condition satisfy the following relations

$$
e_{n} D B_{ \pm}=B_{\mp} e_{n} D, \quad e_{A} e_{a} B_{ \pm}=B_{\mp} e_{A} e_{a} .
$$

where $B_{ \pm}=\frac{1}{2}\left(I \pm e_{n} \omega\right)$ are the projection operators acting on the spinor bundle $\mathbb{S}$. 
Proof. ¿From Lemma 3.2, we get

$$
\begin{aligned}
e_{n} D B_{ \pm} \varphi & =\frac{1}{2} e_{n} D\left(\varphi \pm e_{n} \omega \varphi\right) \\
& =\frac{1}{2} e_{n} D \varphi \pm \frac{1}{2} e_{n} D\left(e_{n} \omega \varphi\right) \\
& =\frac{1}{2} e_{n} D \varphi \pm \frac{1}{2} e_{n} e_{a} \nabla_{a}\left(e_{n} \omega \varphi\right) \\
& =\frac{1}{2} e_{n} D \varphi \pm \frac{1}{2} e_{n} e_{a} e_{n} \nabla_{a}(\omega \varphi) \\
& =\frac{1}{2} e_{n} D \varphi \pm \frac{1}{2} e_{n} e_{a} e_{n} \omega \nabla_{a} \varphi \\
& =\frac{1}{2} e_{n} D \varphi \pm \frac{1}{2} e_{n} \omega e_{a} e_{n} \nabla_{a} \varphi \\
& =\frac{1}{2} e_{n} D \varphi \mp \frac{1}{2} e_{n} \omega e_{n} D \varphi \\
& =B_{\mp} e_{n} D \varphi
\end{aligned}
$$

and

$$
\begin{aligned}
e_{A} e_{a} B_{ \pm} \varphi & =\frac{1}{2} e_{A} e_{a}\left(\varphi \pm e_{n} \omega \varphi\right) \\
& =\frac{1}{2} e_{A} e_{a} \varphi \pm \frac{1}{2} e_{A} e_{a} e_{n} \omega \varphi \\
& =\frac{1}{2}\left(e_{A} e_{a} \varphi \mp e_{n} \omega e_{A} e_{a} \varphi\right) \\
& =B_{\mp} e_{A} e_{a} \varphi .
\end{aligned}
$$

Lemma 3.4. Let $M^{n}$ be a compact spacelike spin submanifold of a pseudoRiemannian manifold $N^{n+m}$. Suppose that the normal bundle of $M$ is spin and $m$ is odd. Let $\widetilde{D}$ be the Dirac-Witten operator on the spinor bundle over M. Denote

$$
\mathbf{R}_{M}=\mu-\varpi_{i A} e_{i} e_{A}+\frac{1}{2} \sum_{\{\alpha, \beta\} \neq\{i, A\} ; \alpha<\beta} \widetilde{R}_{i A \alpha \beta} e_{i} e_{A} e_{\alpha} e_{\beta} .
$$

If $M^{n}$ has a nonempty boundary $\Sigma$, then

$$
\begin{aligned}
\int_{\Sigma}\left\langle e_{n} D \varphi, \varphi\right\rangle-\frac{H}{2}\langle\varphi, \varphi\rangle & +\frac{\operatorname{Tr}\left(\left.p_{A}\right|_{\Sigma}\right)}{2}\left\langle e_{A} e_{n} \varphi, \varphi\right\rangle-\frac{p_{A a n}}{2}\left\langle e_{A} e_{a} \varphi, \varphi\right\rangle \\
& =\int_{M}|\widetilde{\nabla} \varphi|^{2}+\frac{1}{2}\left\langle\mathbf{R}_{M} \varphi, \varphi\right\rangle-|\widetilde{D} \varphi|^{2} .
\end{aligned}
$$

Proof. A straightforward calculation yields

$$
\begin{aligned}
\int_{\Sigma}\left\langle e_{n} \widetilde{D} \varphi, \varphi\right\rangle & =\int_{M}\left\langle\widetilde{D}^{2} \varphi, \varphi\right\rangle-\int_{M}|\widetilde{D} \varphi|^{2} \\
\int_{\Sigma}\left\langle\widetilde{\nabla}_{n} \varphi, \varphi\right\rangle & =-\int_{M}\left\langle p_{A i j} e_{j} e_{A} \widetilde{\nabla}_{i} \varphi, \varphi\right\rangle+\int_{M}\langle\widetilde{\nabla} \widetilde{\nabla} \varphi, \varphi\rangle+\int_{M}|\widetilde{\nabla} \varphi|^{2} .
\end{aligned}
$$


Thus

$$
\begin{aligned}
\int_{M}|\widetilde{D} \varphi|^{2}- & \frac{1}{2}\left\langle\mathbf{R}_{M} \varphi, \varphi\right\rangle-|\widetilde{\nabla} \varphi|^{2} \\
= & -\int_{\Sigma}\left\langle\widetilde{\nabla}_{n} \varphi+e_{n} \widetilde{D} \varphi, \varphi\right\rangle \\
= & -\int_{\Sigma}\left\langle\widetilde{\nabla}_{n} \varphi+e_{n} e_{i} \widetilde{\nabla}_{i} \varphi, \varphi\right\rangle \\
= & -\int_{\Sigma}\left\langle e_{n} e_{a} \widetilde{\nabla}_{a} \varphi, \varphi\right\rangle \\
= & -\int_{\Sigma}\left\langle e_{n} e_{a}\left(\nabla_{a}+\frac{1}{2} h_{a b} e_{n} e_{b}+\frac{1}{2} p_{A a j} e_{j} e_{A}\right) \varphi, \varphi\right\rangle \\
= & -\int_{\Sigma}\left\langle\left(e_{n} D-\frac{1}{2} H\right) \varphi, \varphi\right\rangle-\frac{1}{2} \int_{\Sigma}\left\langle\operatorname{Tr}\left(\left.p_{A}\right|_{\Sigma}\right) e_{A} e_{n} \varphi, \varphi\right\rangle \\
& +\frac{1}{2} \int_{\Sigma}\left\langle p_{A a n} e_{A} e_{a} \varphi, \varphi\right\rangle .
\end{aligned}
$$

Denote by $\vec{P}=\operatorname{Tr}\left(\left.p_{A}\right|_{\Sigma}\right) e_{A}$ the restriction of the mean curvature vector on $\Sigma$. Then $\Sigma$ is a generalized future/past apparent horizon if

$$
H \mp \vec{P} \omega \geq 0
$$

on $\Sigma$ as an endomorphism over $\mathbb{S}$. The physical explanation of this definition relies on the behavior of null geodesics in this case. This will be examined elsewhere.

Theorem 3.5. Let $M^{n}$ be a compact spacelike spin submanifold of a pseudoRiemannian manifold $N^{n+m}$. Suppose that the normal bundle of $M$ is spin and $m$ is odd. Let $\lambda$ be an eigenvalue of the Dirac-Witten operator with a corresponding spinor $\varphi$. If $M^{n}$ has a nonempty boundary $\Sigma$ which is a future or past apparent horizon, and the generalized dominant energy condition (5) holds, then, under the local boundary condition $B_{-} \varphi=0$ for future apparent horizon, or $B_{+} \varphi=0$ for past apparent horizon, we have

$$
\lambda^{2} \geq \frac{n}{2(n-1)} \inf _{M}(\mu-U)
$$

Proof. For the local boundary condition, we have

$$
\int_{\Sigma}\left\langle e_{n} D \varphi, \varphi\right\rangle=0, \quad \int_{\Sigma}\left\langle p_{A a n} e_{A} e_{a} \varphi, \varphi\right\rangle=0 .
$$

The theorem follows by using the modified connection $\widetilde{\nabla}_{i}^{\lambda}=\widetilde{\nabla}_{i}+\frac{\lambda}{n} e_{i}$.

Remark 3.1. If $m=1$, Theorem 2.6 and Theorem 3.5 reduce to the main estimates in [19]. We can also apply the conformal methods in $[12,19]$ to estimate the eigenvalues of the submanifold Dirac-Witten operator. Recall, 
in the case where $m=1$ [19], the Einstein tensors of the conformal metric $\widetilde{\mathbf{g}}=f^{\frac{4}{n-2}} \widetilde{g}$ with $f>0, d f\left(e_{n}\right)=0$ satisfy

$$
\mathbf{T}_{n n}=T_{n n}+\frac{2(n-1)}{n-2} f^{-1} \triangle f, \quad \mathbf{T}_{a n}=T_{a n}+(n-1) h_{a}^{b} \nabla_{b} u
$$

where $f^{\frac{4}{n-2}}=e^{2 u}$ (The authors would like to thank Daniel Maerten who pointed out an error in [19] where the term $h_{a}^{b} \nabla_{b} u$ was missing in the second above formula). For an eigenspinor $\phi$, we define $T_{\phi}^{a}=\left\langle\phi, e_{n} e_{a} \phi\right\rangle /|\phi|^{2}$ on the complement of the zero's set of $\phi$, and by zero on its zero set. Let

$$
L_{\phi}=\frac{4(n-1)}{n-2}\left(\triangle+T_{\phi}^{a} h_{a}^{b} \nabla_{b}\right)+2\left(T_{n n}-\sqrt{\sum_{a} T_{a n}^{2}}\right) .
$$

Then the operator $L$ should be replaced by $L_{\phi}$ in Theorem 3 and Theorem 6 in [19] (It would be interesting to discuss other types of boundary conditions $[14,13,8]$ and to generalize these results to the case $m>1$ ).

\section{Application to gravity}

The Dirac-Witten operator is closely related to the total energy-momentum of spacetimes [26]. A similar result is generalized to 5-dimensional spacetime $N^{4+1}[28,30]$, and to arbitary higher dimensional spacetime $N^{n+1}$ [9]. It is hence natural to study the Dirac-Witten operator in the set-up of pseudo-Riemannian manifolds $N^{n+m}$ with $m \geq 1$.

Let $N^{n+m}$ be a pseudo-Riemannian manifold. Let $M^{n}$ be a spacelike submanifold of $N^{n+m}$ with the induced Riemannian metric denoted by $g$ (instead of $\bar{g}$ for simplicity) and second fundamental form $p_{A}$. An initial data set $\left(M, g, p_{A}\right)$ is asymptotically flat if there is a compact set $K$ such that $M \backslash K$ is the disjoint union of a finite number of subsets $M_{1}, \cdots, M_{l}$ - called the "ends" of $M$ - each diffeomorphic to $\mathbb{R}^{n} \backslash B_{r}^{n}$, where $B_{r}^{n}$ is the closed ball of radius $r$ with center at the coordinate origin. In each end, $g$ and $p_{A}$ satisfy, as $r \rightarrow \infty$,

$$
\begin{gathered}
g_{i j}=\delta_{i j}+O\left(\frac{1}{r^{n-2}}\right), \quad \partial_{k} g_{i j}=O\left(\frac{1}{r^{n-1}}\right), \quad \partial_{l} \partial_{k} g_{i j}=O\left(\frac{1}{r^{n}}\right), \\
p_{A i j}=O\left(\frac{1}{r^{n-1}}\right), \quad \partial_{k} p_{A i j}=O\left(\frac{1}{r^{n}}\right)
\end{gathered}
$$

where $\left\{x^{i}\right\}$ are the Euclidean coordinates of $\mathbb{R}^{n}$. Moreover, the scalar curvature $R$ of $M$ is assumed to be in $L^{1}(M)$.

The ADM total energy $E_{l}$ and the generalized total linear momentum $P_{l k A}$ of the end $M_{l}$ are defined as follows

$$
\begin{aligned}
E_{l} & =\frac{1}{4 \operatorname{Vol}\left(S^{n-1}\right)} \lim _{r \rightarrow \infty} \int_{S_{r}^{n-1}}\left(\partial_{j} g_{i j}-\partial_{i} g_{j j}\right) * d x^{i}, \\
P_{l k A} & =\frac{1}{2 \operatorname{Vol}\left(S^{n-1}\right)} \lim _{r \rightarrow \infty} \int_{\substack{S_{r}^{n-1} \\
14}}\left(p_{A k i}-\delta_{k i} h_{A j j}\right) * d x^{i},
\end{aligned}
$$


where $S_{r}^{n-1}$ is the sphere of radius $r$ in the end and $S^{n-1}$ is the unit sphere in the $n$-dimensional Euclidean space. The generalized total linear momentum $P_{l}$ of the end $M_{l}$ is actually a map from $\mathbb{R}^{n} \times \mathbb{R}^{m}$ to $\mathbb{R}$, with the components $P_{l k A}$ where $1 \leq k \leq n, n+1 \leq A \leq n+m$. It is therefore a bi-vector. Now we can prove the following generalized positive mass theorem.

Theorem 4.1. Let $M^{n}$ be a compact spacelike spin submanifold of a pseudoRiemannian manifold $N^{n+m}$, which has possibly finite number of generalized future/past apparent horizons $\Sigma_{i}$. Suppose that the normal bundle of $M$ is spin and $m$ is odd. If the generalized dominant energy condition (5) holds, then

$$
E_{l} \geq \sqrt{\sum_{k, A} P_{l k A}^{2}} .
$$

That $E_{l_{0}}=0$ for some end $M_{l_{0}}$ implies that $M$ has only one end and $\widetilde{R}_{i j \alpha \beta}=$ 0 over $M$.

Proof. Let $\breve{\varphi}$ be any constant spinor on the flat space $\mathbb{R}^{n+m}$ with unit norm under the positive definite spinor inner product. Then we can solve the boundary value problem for the Dirac-Witten operator on $M$ using the same argument as that in $[26,21,3,28,29,30,9]$. Let $\varphi$ be such a solution with the boundary value $\breve{\varphi}$ at infinity on the end $M_{l}$, and zero at infinity on the other ends. Denote $\partial_{\alpha}$ by $\breve{e}_{\alpha}$. The formula (4) implies that

$$
\begin{aligned}
\int_{M}|\widetilde{\nabla} \varphi|^{2} & \leq \int_{S_{\infty}^{n-1}}\left\langle\breve{\varphi}, \sum_{i \neq j} \breve{e}_{i} \breve{e}_{j} \widetilde{\nabla}_{j} \breve{\varphi}\right\rangle * d x^{i} \\
& =4 E_{l}+\int_{S_{\infty}^{n-1}}\left\langle\varphi, \frac{1}{2} \sum_{i \neq j} p_{A j k} \breve{e}_{i} \breve{e}_{j} \breve{e}_{k} \breve{e}_{A} \breve{\varphi}\right\rangle * d x^{i} \\
& =4 E_{l}+\int_{S_{\infty}^{n-1}}\left\langle\varphi, \frac{1}{2}\left(p_{A i k}-\delta_{i k} p_{A j j}\right) \breve{e}_{k} \breve{e}_{A} \breve{\varphi}\right\rangle * d x^{i} \\
& =4 E_{l}+4\left\langle\breve{\varphi}, P_{l k A} \breve{e}_{k} \breve{e}_{A} \breve{\varphi}\right\rangle .
\end{aligned}
$$

Then (15) follows by choosing $\breve{\varphi}$ such that $P_{l k A} \breve{e}_{k} \breve{e}_{A} \breve{\varphi}=-\sqrt{\sum_{k, A} P_{l k A}^{2}} \breve{\varphi}$.

If $E_{l_{0}}=0$, then for any constant spinor $\breve{\varphi}$, we have $\widetilde{\nabla}_{i} \varphi=0$ for solution of the Dirac-Witten equation with the boundary value $\breve{\varphi}$ at infinity on the end $M_{l_{0}}$, and zero at infinity on the other ends. This implies that $M$ has only one end and $\widetilde{R}_{i j \alpha \beta}=0$ over $M$.

Acknowledgement: This work was partially done while X. Zhang visited School of Mathematics and Statistics, University of Sydney and Institut Élie Cartan Université Henri Poincaré, Nancy 1, and he would like to thank them for their hospitality. He would also like to thank R.B. Zhang for many useful conversations. Partial financial support from National Science Foundation 
of China (grants 10725105, 10731080, 11021091) and Chinese Academy of Sciences is gratefully acknowledged.

\section{REFERENCES}

[1] W. Ballmann, J. Bruning, G. Carron, Regularity and index theory for DiracSchrödinger systems with Lipschitz coefficients, J. Math. Pures Appl. 89, 429476 (2008).

[2] C. Bär, Extrinsic bounds of the Dirac operator, Ann. Glob. Anal. Geom. 16, 573-596 (1998).

[3] R. Bartnik, The mass of an asymptotically flat manifold, Comm. Pure Appl. Math. 36, 661-693 (1986).

[4] R. Bartnik, P.T. Chrusćiel, Boundary value problems for Dirac-type equations, J. reine angew. Math. 579, 1373 (2005).

[5] H. Baum, Spin-Strukturen und Dirac-Operatoren über pseudo-Riemannsche Mannigfaltigkeiten, Teubner-Verlag, Stuttgart/Leipzig, Band 41 (1981).

[6] H. Baum, A remark on the spectrum of the Dirac operator on pseudo-Riemannian spin manifolds, preprint, 1996.

[7] B. Booß-Bavnvek, K.P. Wojciechoski, Elliptic boundatry problems for Dirac operators, Birkhäuser, Boston, 1993.

[8] D. Chen, Eigenvalue estimates for the Dirac operator with generalized APS boundary condition, J. Geom. Phys. 57, 379-386 (2007).

[9] L. Ding, Positive mass theorems for higher dimensional Lorentzian manifolds, J. Math. Phys. 49, 022504 (2008).

[10] T. Friedrich, Der erste Eigenwert des Dirac-Operators einer kompakten Riemannschen Mannifaltigkeit nicht negativer Skalarkrümmung, Math. Nach. 97, 117-146 (1980).

[11] N. Ginoux, B. Morel, Eigenvalue estimates for the submanifold Dirac operator, Int. J. Math. 13, 533-548 (2002).

[12] O. Hijazi, A conformal lower bound for the smallest eigenvalue of the Dirac operator and killing spinors, Commun. Math. Phys. 104, 151-162 (1986).

[13] O. Hijazi, S. Montiel, A. Roldán, Eigenvalue boundary problem for the Dirac operator, Commun. Math. Phys. 231, 375-390 (2002).

[14] O. Hijazi, S. Montiel, X. Zhang, Eigenvalues of the Dirac operator on manifolds with boundary, Commun. Math. Phys. 221, 255-265 (2001).

[15] O. Hijazi, S. Montiel, X. Zhang, Conformal lower bounds for the Dirac operator of embedded hypersurfaces, Asian J. Math. 6, 23-36 (2002).

[16] L. Hörmander, The Analysis of Linear Partial Differential Operators III. Berlin, Springer, 1985.

[17] O. Hijazi, X. Zhang, Lower bounds for the eigenvalues of the Dirac operator, Part I. the hypersuface Dirac operator, Ann. Global Anal. Geom. 19, 355-376 (2001).

[18] O. Hijazi, X. Zhang, Lower bounds for the eigenvalues of the Dirac operator, Part II. The submanifold Dirac operator, Ann. Global Anal. Geom., 19, 163-181 (2001).

[19] O. Hijazi, X. Zhang, The Dirac-Witten operator on spacelike hypersurfaces, Comm. Anal. Geom. 11, 737-750 (2003).

[20] B. Morel, Eigenvalue estimates for the Dirac-Schrödinger operators, J. Geom. Phys. 38, 1-18 (2001).

[21] T. Parker, C. Taubes, On Witten's proof of the positive energy theorem, Commun. Math. Phys. 84, 223-238(1982).

[22] R.T. Seeley, Complex powers of an elliptic operator, Proc. Sympos. Pure. Math. 10, 288-307 (1967).

[23] R. Schoen, S.T. Yau, On the proof of the positive mass conjecture in general relativity, Commun. Math. Phys. 65, 45-76 (1979). 
[24] R. Schoen, S.T. Yau, The energy and the linear momentum of spacetimes in general relativity, Commun. Math. Phys. 79, 47-51 (1981).

[25] R. Schoen, S.T. Yau, Proof of the positive mass theorem II, Commun. Math. Phys. 79, 231-260 (1981).

[26] E. Witten, A new proof of the positive energy theorem, Commun. Math. Phys. 80, 381-402 (1981).

[27] X. Zhang, Lower bounds for eigenvalues of hypersurface Dirac operators, Math. Res. Lett. 5, 199-210(1998); A remark: Lower bounds for eigenvalues of hypersurface Dirac operators, Math. Res. Lett. 6, 465-466 (1999).

[28] X. Zhang, Positive mass conjecture for five-dimensional Lorentzian manifolds, J. Math. Phys. 40, 3540-3552 (1999).

[29] X. Zhang, Angular momentum and positive mass theorem, Commun. Math. Phys. 206, 137-155 (1999).

[30] X. Zhang, Positive mass theorem for hypersurface in 5-dimensional Lorentzian manifolds, Comm. Anal. Geom. 8, 635-652 (2000).

Department of Mathematical Sciences, Tsinghua University, Beijing, 100084, P.R. China

E-mail address: dgchen@math.tsinghua.edu.cn

Institut Élie Cartan Université Henri Poincaré, NAncy 1, B.P. 239, 54506 VAndoeuvre-LÈs-NANCY CEDex, FRANCE

E-mail address: hijazi@iecn.u-nancy.fr

Institute of Mathematics, Academy of Mathematics and Systems Science, Chinese Academy of Sciences, Beijing 100190, China

E-mail address: xzhang@amss.ac.cn 